\title{
Nanoparticles: Key to Optimization of Drug Therapy
}

\section{Onyeji CO*}

Faculty of Pharmacy, Obafemi Awolowo University, Nigeria

*Corresponding author: Cyprian Ogbonna Onyeji, Faculty of Pharmacy, Obafemi

Awolowo University, Ile-Ife, Nigeria, Tel: +2348037058720; Email:

conyeji@oauife.edu.ng

\section{Editorial}

The application of nano-sized particles (usually less than $200 \mathrm{~nm}$ ) in pharmacology and medicine is a young interdisciplinary science that started receiving active research in the $20^{\text {th }}$ century. Nanoparticles have various applications in nanotherapy, nanodiagnostics and theranostics. Nanotherapy and nanodiagnostics, as the words suggest, are the applications of nanoparticles in therapy and diagnosis, respectively, while theranostics involves using nanoparticles which combine specific targeted therapy based on specific targeted diagnostic tests. Generally, research in the use of nanoparticles in nanotherapy is concentrated on investigation of the possibilities of manipulating drug delivery systems for targeted transport of drug molecules or active substances for optimization of therapy. It has been asserted that since physiological processes at cellular and subcellular levels occur on a nanoscale, there is a very great potential in the application of nanoparticles for improving medical diagnostics and therapeutics [1].

The first nanoparticles used for targeted drug therapy was developed at the end of the 1960s and this finding has been followed by rigorous research into the development of diverse carrier systems applied for optimal delivery of active agents [2]. Thus, cell-specific targeting can be accomplished by attaching drugs to specially designed carriers. Various nanostructures have been tested as carriers in targeted drug delivery systems and these include: (a) Liposomes (b) Natural polymers and derivatives, (c) Synthetic Polymers, (d) Dendrimers (e) Solid-lipid formulations; (f) Silica-based nanoparticles; and (g) Magnetic nanoparticles.

\section{Liposomes}

Liposomes are spherically-shaped vesicles consisting of one or more phospholipid bilayers surrounding aqueous phases, where the polar head groups of the phospholipids are oriented towards the aqueous compartments. Phospholipids are known to automatically form closed structures when they are hydrated in aqueous solutions. Such vesicles can transport hydrophilic or hydrophobic drugs which are entrapped in the aqueous compartment or lipid layers of the liposome. Generally, particle sizes of liposomes range from $30 \mathrm{~nm}$ to several micrometers and they are classified on the basis of their size and number of bilayers. They can be classified into one of two categories: multilamellar vesicles (MLV, with several lipid bilayers) and unilamellar vesicles (with one lipid bilayer).The unilamellar vesicles can also be classified into either large unilamellar vesicles (LUV) or small unilamellar vesicles (SUV). The versatility of liposomes makes them highly valuable for various therapeutic applications in immunology, tumor therapy, gene delivery, antiviral therapy as well as to deliver drugs and proteins. We and several other authors have demonstrated that drug encapsulation through liposomal formulation or other drug delivery systems can lead to improved efficacy and therapeutic index, and reduced toxicity of a variety of drugs [2-4].

\section{Polymer}

Examples of natural polymers and derivatives used in formulating drug delivery systems include Chitosan, Dextrane, Gelatin, Alginates, Albumin, and Starch. Also, there is a wide range of synthetic polymers such as poly(2-hydroxyethyl methacrylate), poly-(Nvinylpyrrolidone), poly-(methylmethacrylate), pol-(vinyl alcohol),polyacrylamide, polycarprolactone, poly-alkylcyanoacrylates and polylactic acid. Considerable research efforts have been directed towards developing safe and efficient natural and synthetic polymer-based nanoparticles as carriers for a wide range of drugs for therapeutic applications. Their chemically modified derivatives are also used in delivering the bioactive 


\section{Advances in Pharmacology and Clinical Trials}

molecules. Using both natural and synthetic polymers, several hydrogel nanoparticulate systems have been prepared and characterized in recent years. Hydrogel nanoparticles have gained considerable attention as one of the most promising nanoparticulate drug delivery systems owing to their unique potentials of combining the capacity to encapsulated drugs with the characteristics of a hydrogel system (e.g. hydrophilicity and extremely high water content).

\section{Dendrimers}

These are nano-sized repetitively branched polymeric structures that are typically symmetric around the core, and they often adopt a spherical morphology. Due to the physical characteristics of dendrimers which include the ability to encapsulate compounds and possession of large number of peripheral groups that can be functionalized, this class of branched polymers have been evaluated as nanostructures for targeted drug delivery.

\section{Solid Lipid Formulations}

These are nanoparticulate drug delivery systems that have been introduced after liposomes and polymeric systems. They are composed of a single lipid core matrix that is solid at body temperature, and is coated with a surfactant acting as a stabilizer. The lipids used are biodegradable and are generally recognized as safe. This nanostructure is efficient as carriers for poorly watersoluble pharmaceutical agents. They represent an alternative carrier system to liposomes and polymeric nanoparticles as they have the advantages of these traditional systems but avoid some of their major drawbacks. The lipid matrix of solid lipid nanoparticles is made from physiological lipids and this offers a clear advantage since the danger of toxicity is minimal.

\section{Silica-Based Nanoparticles}

This class of nanoparticles especially the mesoporous form is widely used as drug carriers. This is because silica possesses favourable properties such as their specific surface characteristics, porosity, biocompatibility and capacity for functionalization which render them useful for providing drug delivery. The unique mesoporous structure of silica facilitates effective loading of drugs.

\section{Magnetic Nanoparticles}

These consist of porous polymers that contain magnetic materials precipitated within the pores. Several inorganic materials with magnetic properties may be chosen but for several reasons, the iron oxides are the preferred. The potential of magnetic nanoparticle as drug delivery system is attributable to the intrinsic properties of their magnetic cores combined with their drug loading capability. Also, additional biochemical properties can be imparted on them by means of a suitable coating material. Due to these properties therapeutic agents can be attached to, or encapsulated within the nanostructures.

Generally, modalities for enhancing drug delivery specificity of nanoparticles include a multifunctional approach. Targeting and drug delivery to specific tissues can be optimized by modification of different characteristics and properties of the nanostructures such composition of the material, surface charge, size, and the method of preparation. Also, surfaces of the nanoparticles can be individually modified for specific targeted transports by being coupled with site-specific ligands (for example antibodies, proteins, peptides, carbohydrates etc.) which deliver the entrapped agent to the intended site of action [4]. Other approaches include attachment of stealth-like features to enable the nanoparticles evade the immune system and prevent opsonization, improvement of cell uptake by attaching membrane-permeation moieties and modulation of drug release at intended site of action through incorporation of microenvironment sensors that can trigger drug release [5].

In conclusion, nanoparticulate drug delivery systems have been applied to several therapeutic agents. Literature abounds with studies aimed at producing nanoparticulate drug delivery systems with reduced drug toxicities and enhanced efficacy compared with free complements, while ensuring the safety and biocompatibility of the nanoparticle materials. However, despite these potential advantages, only a relatively small number of nanoparticle-based medicines have been approved for clinical use. A challenge to extensive deployment of these drug delivery systems in therapeutics remains the consideration of benefits versus costs of production, as well as stability and safety profiles of the formulations.

\section{References}

1. Moghimi SM, Hunter AC, Murray JC (2005) Nanomedicine: Current status and future prospects. FASEB J 19(3): 311-330.

2. Krukemeyer MG, Krenn V, Huebner F, Wagner W, Resch R (2015) History and Possible Uses of Nanomedicine Based on Nanoparticles and 


\section{Advances in Pharmacology and Clinical Trials}

Nanotechnological Progress. J Nanomed Nanotechnol 6(6): 1-7.

3. Onyeji CO, Nightingale $\mathrm{CH}$, Nicolau DH, Quintiliani R (1994) Efficacies of Liposome-Encapsulated Clarithromycin and Ofloxacin against Mycobacterium avium-M. intracellulare Complex in Human Macrophages. Antimicrob Agents Chemother 38(3): 523-527.
4. Akbarzadeh A, Rezaei-Sadabady R, Davaran S, Joo SW, Zarghami N, et al. (2013) Liposome: classification, preparation, and applications. Nanoscale Research Letters 8(1): 102.

5. Cheng Z, Al-Zaki A, Hui JZ, Muzykantov VR, Tsourkas A (2012) Multifunctional nanoparticles: cost versus benefit of adding targeting and imaging capabilities. Science 338: 903-910. 